\title{
Synthesis and Structures of Oxo-Bridged Distannyl- and Digermyldirhenium Complexes
}

\author{
Richard D. Adams, ${ }^{*}$ Burjor Captain, Carl B. Hollandsworth, Mikael Johansson, and \\ Jack L. Smith, Jr. \\ Department of Chemistry and Biochemistry \\ University of South Carolina \\ Columbia, SC, 29208
}

\section{Details on the DFT Calculations}

Density functional theory calculations were performed using the Amsterdam Density Functional (ADF) program, version 2004.01, developed by Baerends et al. ${ }^{1}$ and vectorized by Ravenek. ${ }^{2}$ The numerical integration scheme used was developed by te Velde et al., ${ }^{3}$ and the geometry optimization procedure was based on the method of Verslius and Ziegler. ${ }^{4}$ Geometry optimizations were carried out with no symmetry restrictions using the local exchange-correlation potential of Vosko et al. ${ }^{5}$ and the nonlocal exchange and correlation corrections of Perdew and Wang (PW91). ${ }^{6}$ For rhenium, the electronic configuration was described using a double- $\zeta$ STO basis for $5 \mathrm{~s}$ and a triple$\zeta$ for $6 \mathrm{~s}, 5 \mathrm{p}, 5 \mathrm{~d}$, and $4 \mathrm{f}$, augmented by a $6 \mathrm{p}$ and a $5 \mathrm{f}$ polarization function. The core was frozen and was defined to be $4 \mathrm{~d}$ and below. For germanium, the electronic configuration was described using a triple- $\zeta$ STO basis for $4 \mathrm{~s}, 3 \mathrm{~d}$, and $4 \mathrm{p}$ with the core frozen below $3 \mathrm{p}$. For oxygen and carbon, a triple- $\zeta$ STO basis was used for the valence $2 \mathrm{~s}$ and $2 \mathrm{p}$ orbitals, augmented by a $3 \mathrm{~d}$ and a $4 \mathrm{f}$ polarization function, with the core 1 s frozen. Hydrogen was

\footnotetext{
* Author to whom correspondence should be addressed. E-mail: adams@mail.chem.sc.edu. Tel: 803$777-7187$.
} 
also treated using a triple- $\zeta$ STO basis for its $1 \mathrm{~s}$, and was augmented with a $2 \mathrm{p}$ and a $3 \mathrm{~d}$ polarization function. All atoms were also corrected for relativistic effects using the zeroth order regular approximation (ZORA) ${ }^{7}$ method. All accuracy and convergence parameters were left at the default level, with the exception of the general Integration accuracy, which was set to 7.0.

\section{$\underline{\text { References }}$}

1. (a) Baerends, E. J.; Ellis, D.E.; Ros, P. Chem. Phys. 1973, 2, 41. (b) Baerends, E. J.; Ros, P. Chem. Phys. 1973, 2, 52. (c) te Velde, G.; Bearends, E. J. J. Comput. Phys. 1992, 92, 84. (d) Fonseca, C. G.; Visser, O.; Snijders, J. G.; te Velde, G.; Baerends, E. J. In Methods and Techniques in Computational Chemistry, METECC-95; Clementi, E., Corongiu, G., Eds.; STEF: Cagliara, Italy, 1995; p. 305.

2. Ravenek, W. In Algorithms and Applications on Vector and Parallel Computers; te Riele, H. J. J., Dekker, T. J., van de Horst, H. A, Eds.; Elsevier: Amsterdam, The Netherlands, 1987.

3. (a) te Velde, G.; Baerends, E. J. J. Comput. Chem. 1992, 99, 84. (b) Boerrigter, P. M.; te Velde, G.; Baerends, E. J. Int. J. Quantum Chem. 1998, 33, 87.

4. Versluis, L.; Ziegler, T. J. Chem. Phys. 1988, 88, 322.

5. Vosko, S. H.; Wilk, L.; Nusair, M. Can. J. Phys. 1980, 58, 1200.

6. Perdew, J. P. Phys. Rev. B 1992, 46, 6671.

7. van Lenthe, E.; Ehlers, A. E.; Baerends, E. J. J. Chem. Phys. 1999, 110, 8943.

\section{$\underline{\text { Introduction }}$}

The electronic structure of $\mathrm{Re}_{2}(\mathrm{H})(\mathrm{CO})_{8}\left(\mathrm{Ph}_{2} \mathrm{GeOHGePh}_{2}\right)$ along with its anionic conjugate base were calculated via DFT (ADF 2004.01, PW91). The conjugate base is formed via deprotonation of the $\mathrm{O}-\mathrm{H}$ bridge between the Ge atoms (in contrast to removal of the hydride ligand bridging Re). This study was undertaken in an effort to correlate 
changes in the bonding within Re-Ge-O-Ge-Re ring system upon deprotonation with changes in the molecular orbital manifold of the cluster.

\section{Geometry Optimization}

Geometry optimization was carried out on both the neutral cluster and its uninegative conjugate base. The crystal structure coordinates for the neutral cluster were used for the starting geometry of both optimizations. Although the neutral compound has pseudo- $C_{2}$ symmetry in the solid state, the geometry optimizations were performed without symmetry restraints. Corrections for relativistic effects using the ZORA approximation were also used, as specified in the computational methods section. The coordinates for the anionic cluster were generated by simply removing the hydrogen atom bound to oxygen from the input coordinate list. Views along 3 axes for the optimized geometries for both clusters are shown in Fig. 1. Selected calculated bonding parameters for both clusters are presented in Table 1. 

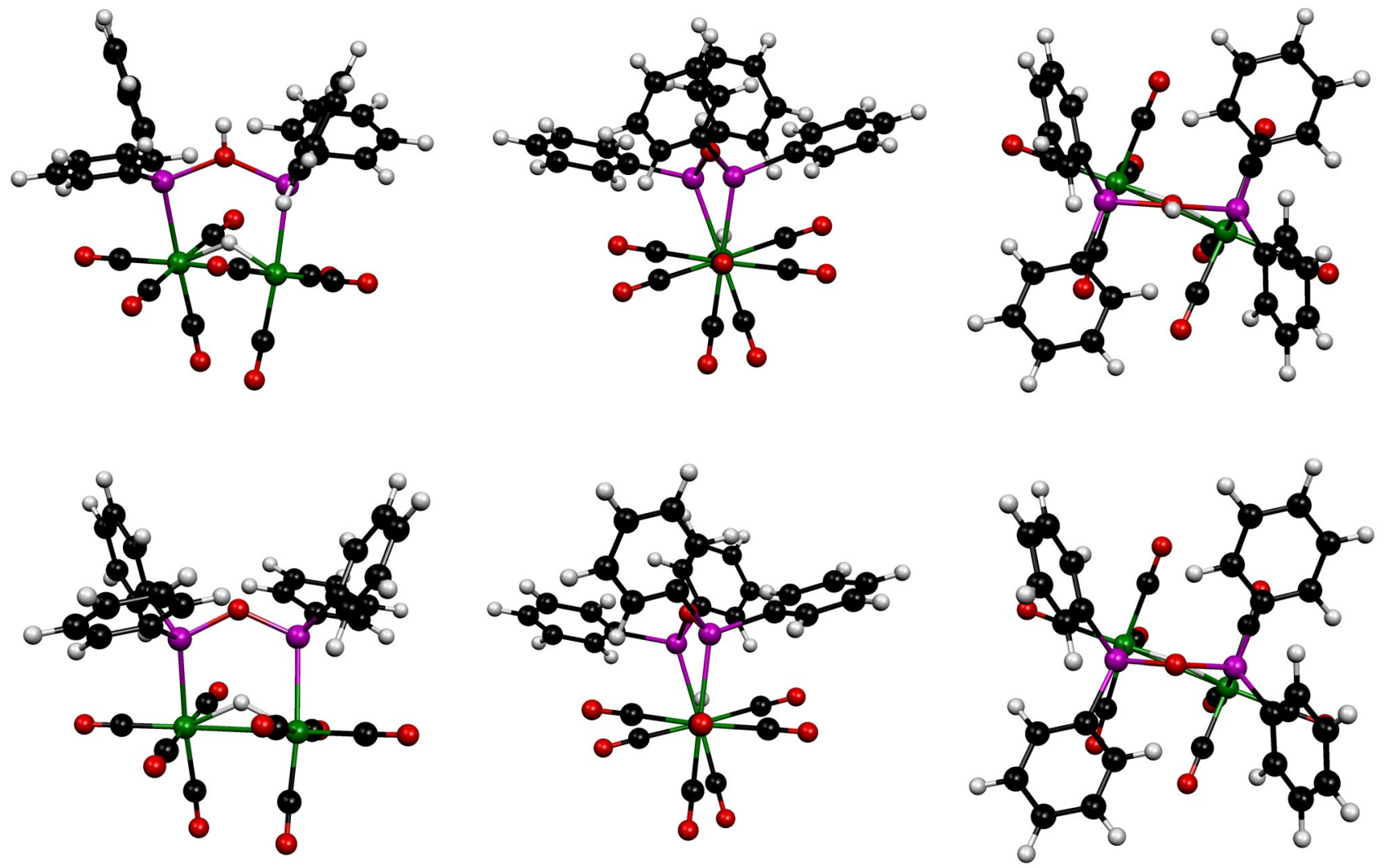

Figure 1. Axial views of the converged geometries of the neutral cluster (top) and its anionic conjugate base (bottom). 


$$
\mathrm{Re}_{2}(\mathrm{H})(\mathrm{CO})_{8}\left(\mathrm{Ph}_{2} \mathrm{GeOHGePh}_{2}\right) \quad\left[\mathrm{Re}_{2}(\mathrm{H})(\mathrm{CO})_{8}\left(\mathrm{Ph}_{2} \mathrm{GeOGePh}_{2}\right)\right]^{-1}
$$

\begin{tabular}{|c|c|c|c|c|c|c|}
\hline Re-Re & 3.324 & & & 3.315 & & \\
\hline Re-Ge & 2.586 & & & 2.643 & & \\
\hline $\mathrm{Re}-\mathrm{Ge}$ & 2.586 & & & 2.649 & & \\
\hline Ge-O & 2.003 & & & 1.833 & & \\
\hline $\mathrm{Ge}-\mathrm{O}$ & 1.994 & & & 1.826 & & \\
\hline Re-H & 1.819 & & & 1.823 & & \\
\hline Re-H & 1.825 & & & 1.820 & & \\
\hline \multirow[t]{2}{*}{$\mathrm{O}-\mathrm{H}$} & 0.982 & & & N/A & & \\
\hline & $\mathrm{Re}-\mathrm{C}$ & $\mathrm{C}-\mathrm{O}$ & $\mathrm{Re}-\mathrm{C}-\mathrm{O}$ & $\mathrm{Re}-\mathrm{C}$ & $\mathrm{C}-\mathrm{O}$ & $\mathrm{Re}-\mathrm{C}-\mathrm{O}$ \\
\hline $\mathrm{Re}-\mathrm{CO}_{\mathrm{eq}}$ & 1.991 & 1.155 & 178.6 & 1.988 & 1.158 & 178.8 \\
\hline $\mathrm{Re}-\mathrm{CO}_{\mathrm{ax}}$ & 1.943 & 1.161 & 179.2 & 1.931 & 1.167 & 178.9 \\
\hline $\mathrm{Re}-\mathrm{CO}_{\mathrm{eq}}$ & 1.997 & 1.153 & 179.3 & 1.990 & 1.156 & 178.4 \\
\hline $\mathrm{Re}-\mathrm{CO}_{\mathrm{ap}}$ & 1.962 & 1.158 & 178.0 & 1.954 & 1.164 & 177.3 \\
\hline $\mathrm{Re}-\mathrm{CO}_{\mathrm{eq}}$ & 1.998 & 1.153 & 179.8 & 1.989 & 1.156 & 178.9 \\
\hline $\mathrm{Re}-\mathrm{CO}_{\mathrm{eq}}$ & 1.990 & 1.155 & 179.8 & 1.985 & 1.158 & 178.6 \\
\hline $\mathrm{Re}-\mathrm{CO}_{\mathrm{ax}}$ & 1.938 & 1.161 & 178.1 & 1.932 & 1.166 & 178.7 \\
\hline $\mathrm{Re}-\mathrm{CO}_{\mathrm{ap}}$ & 1.967 & 1.158 & 178.3 & 1.954 & 1.164 & 177.4 \\
\hline $\mathrm{Re}-\mathrm{Re}-\mathrm{Ge}$ & 92.0 & & & 88.1 & & \\
\hline $\mathrm{Re}-\mathrm{Re}-\mathrm{Ge}$ & 91.0 & & & 87.8 & & \\
\hline $\mathrm{Re}-\mathrm{Ge}-\mathrm{O}$ & 105.8 & & & 113.8 & & \\
\hline Re-Ge-O & 106.5 & & & 113.3 & & \\
\hline Re-H-Re & 131.6 & & & 131.0 & & \\
\hline Ge-O-Ge & 134.1 & & & 128.4 & & \\
\hline Ge-O-H & 111.8 & & & N/A & & \\
\hline $\mathrm{Ge}-\mathrm{O}-\mathrm{H}$ & 113.7 & & & N/A & & \\
\hline
\end{tabular}

Table 1. Calculated bonding parameters for the neutral cluster (left) and its anionic conjugate base (right). Bond distances are in $\AA$ and angles are ${ }^{\circ}$. 


\section{Geometry Optimization Results}

Upon removal of a proton from the neutral cluster, three changes in the Re-Ge-OGe-Re ring system are observed. The Re-Re bond distance is predicted to shorten very slightly by approx. $0.01 \AA$, the Ge-O bond distances are predicted to shorten by approx. $0.2 \AA$, and the Re-Ge bond distances are predicted to elongate by $0.06 \AA$. The most pronounced change is in the $\mathrm{Ge}-\mathrm{O}$ bond distance. The $\mathrm{Re}-\mathrm{Ge}-\mathrm{O}-\mathrm{Ge}-\mathrm{Re}$ ring angles are predicted to change slightly upon deprotonation with the most pronounced change in angle being the ca. $8^{\circ}$ opening of the Re-Ge-O angle. Such an opening is accompanied and surely helped along by the lengthening of the Re-Ge bonds. One can envision this as the entire $\mathrm{Ph}_{2} \mathrm{GeOGePh}_{2}$ bridging ligand moving away from the dirhenium moiety and opening slighly.

Upon deprotonation, all of the Re-C bond distances are predicted to shorten slightly, and this is accompanied by lengthening of the C-O bond distances. [Have Jack check to see that this is supported by IR]. Lessening of the Re-Ge bonding thus allows for greater Re-to-carbonyl backbonding.

\section{$\underline{\text { Molecular Orbitals }}$}

The electronic structures of the neutral and anionic clusters were examined by performing a single point energy calculation on the respective converged geometries. The results of these studies are summarized in Tables 2 and 3. 
Table 2. Molecular orbital pictures and major contributions for the complex 4.

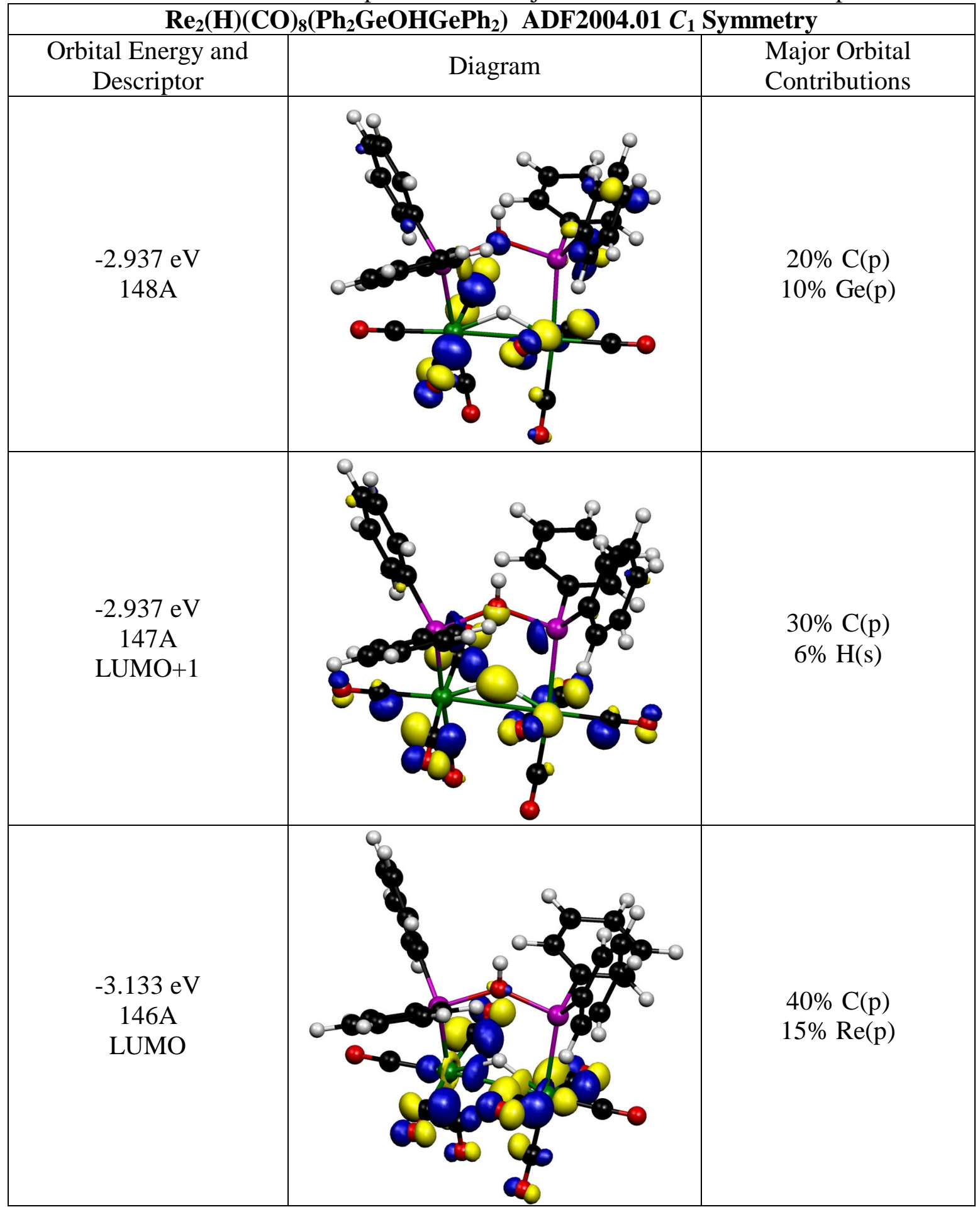




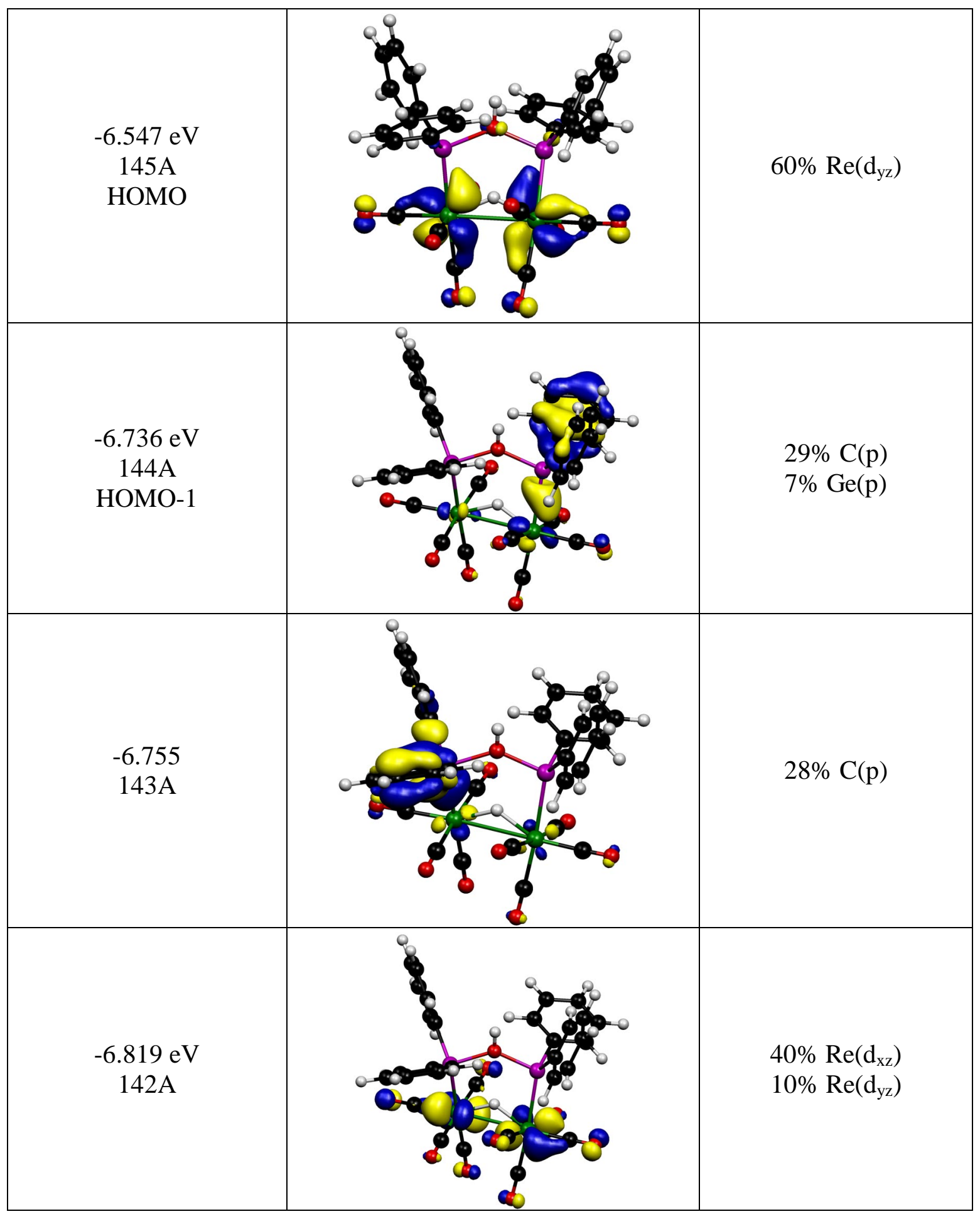




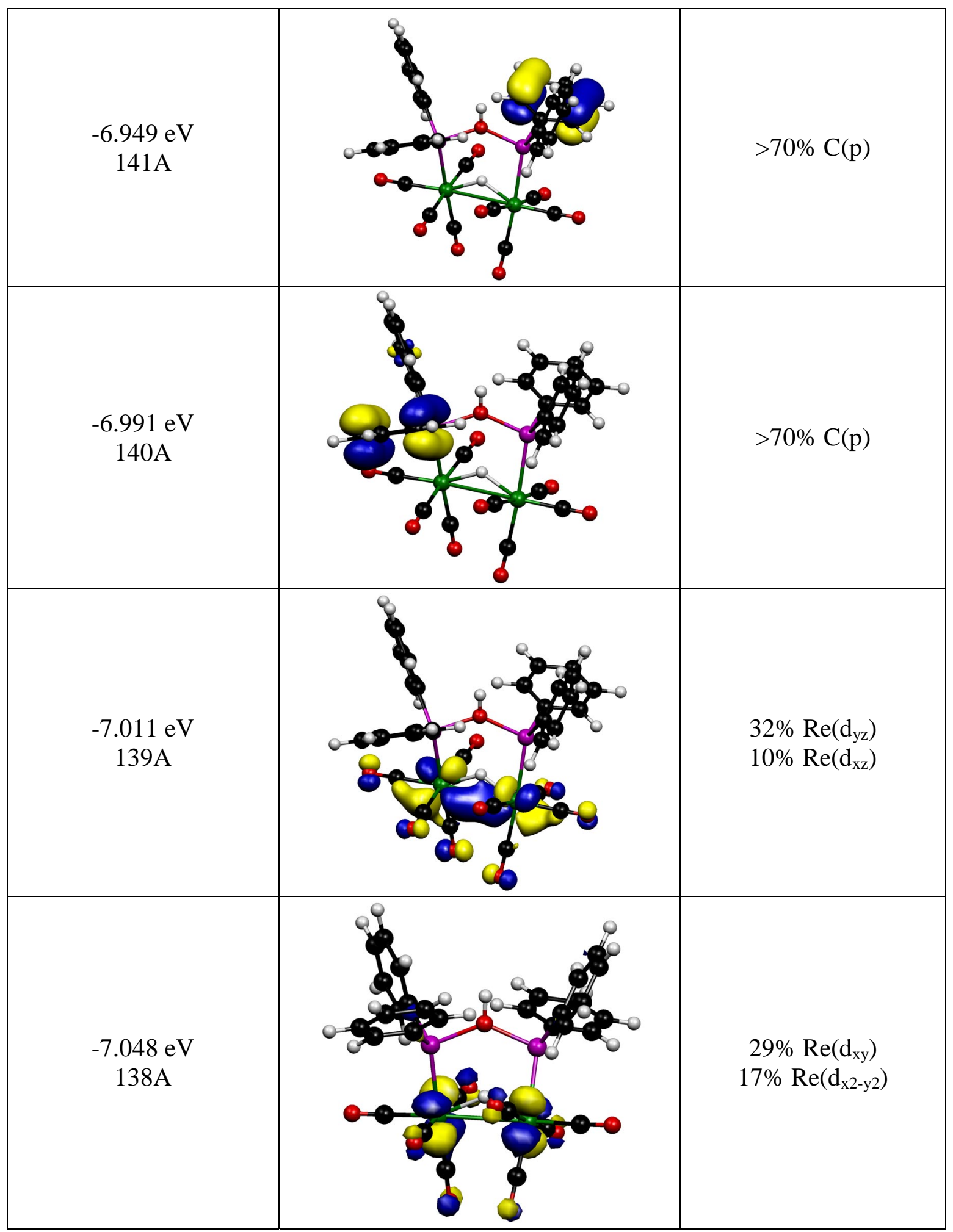




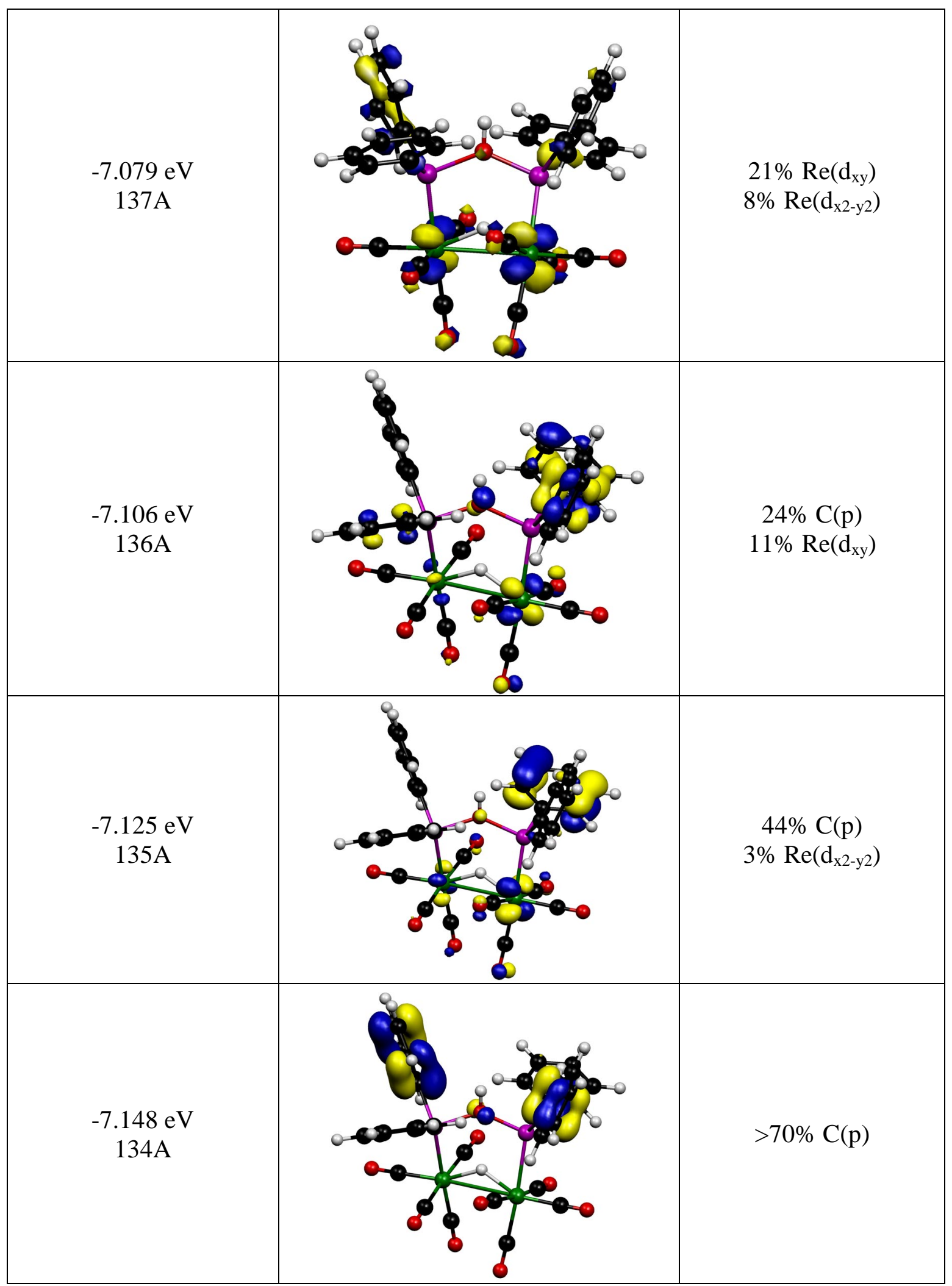




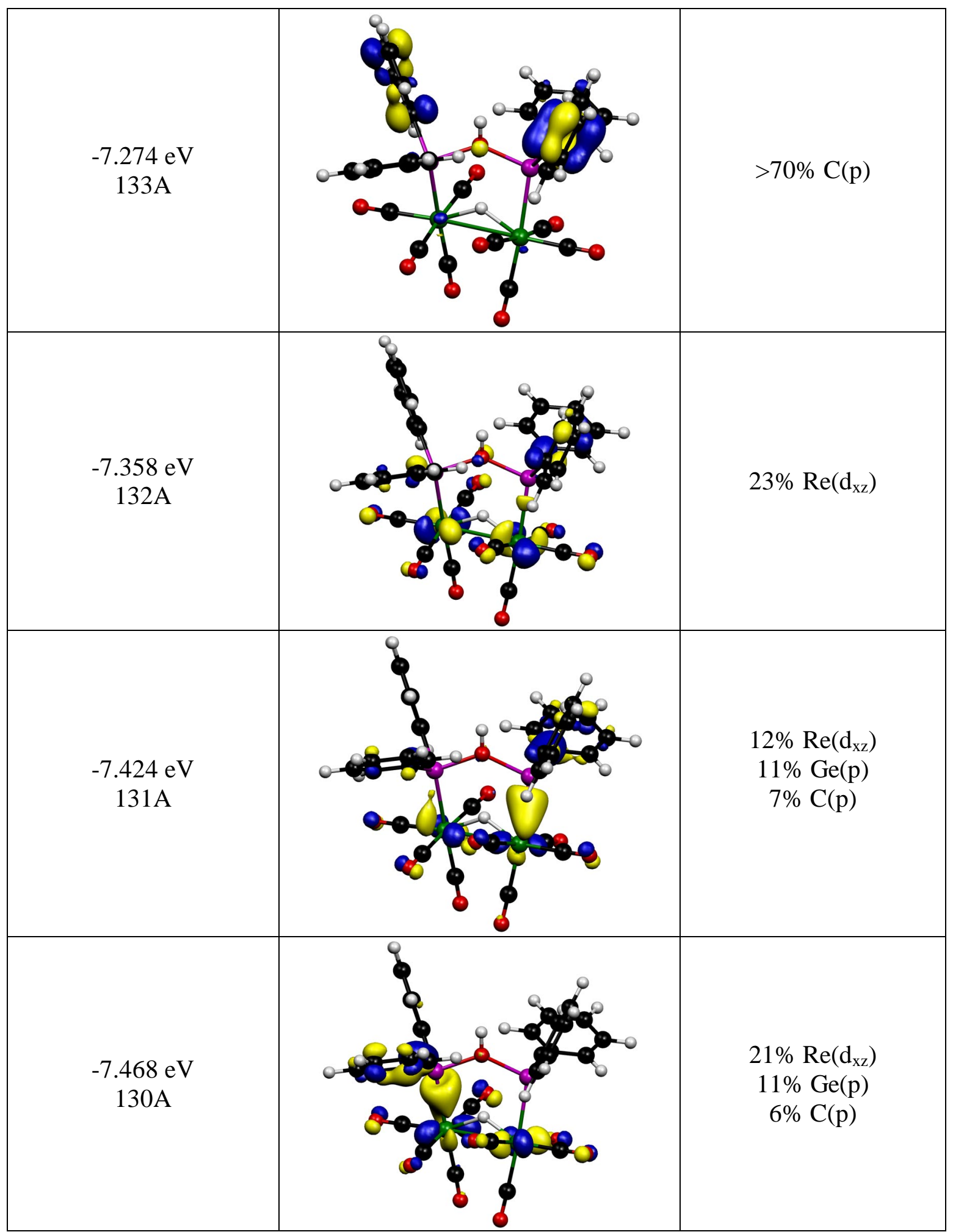




-7.785 eV


Table 3. Molecular orbital pictures and major contributions for the anionic cluster.

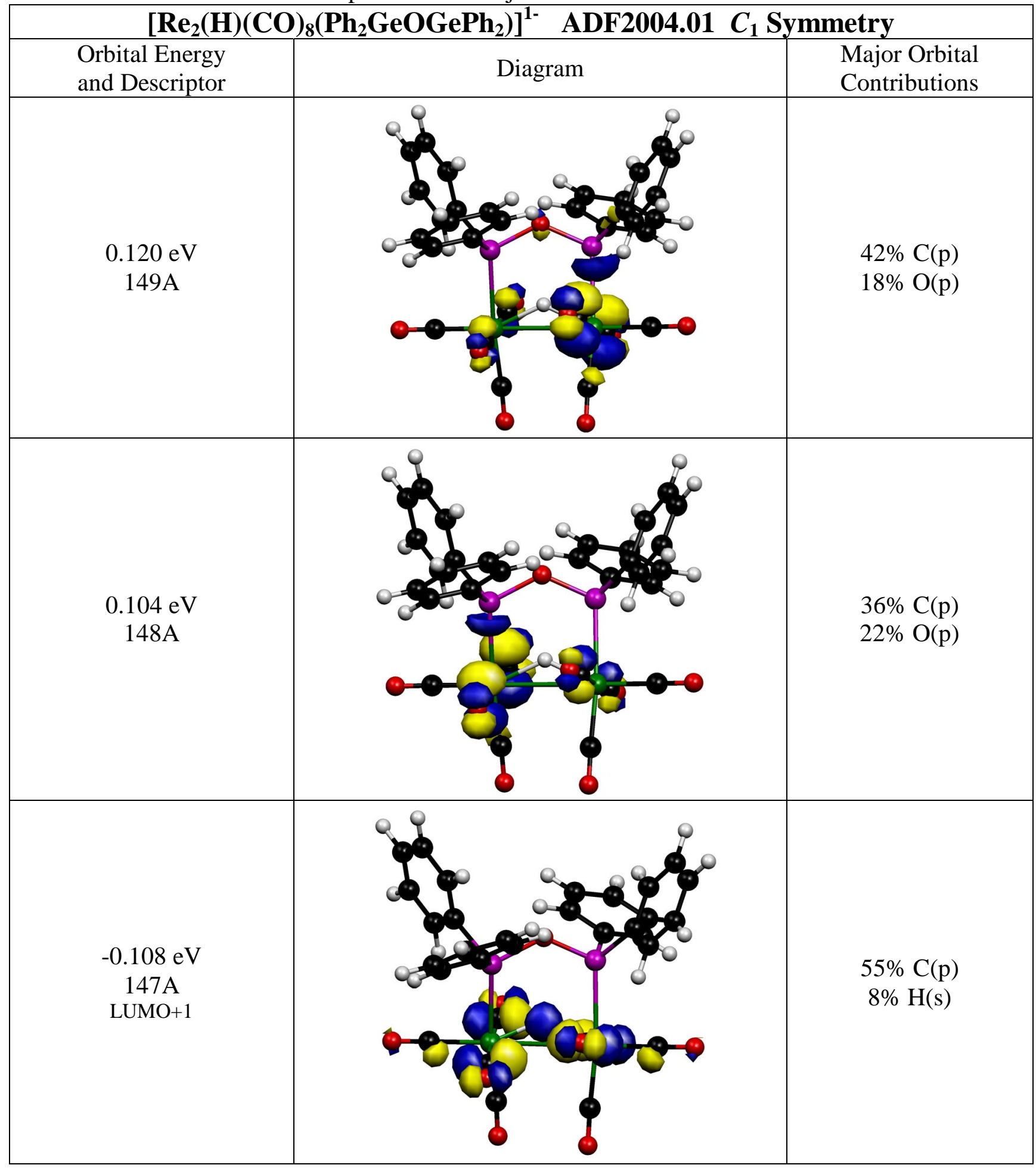




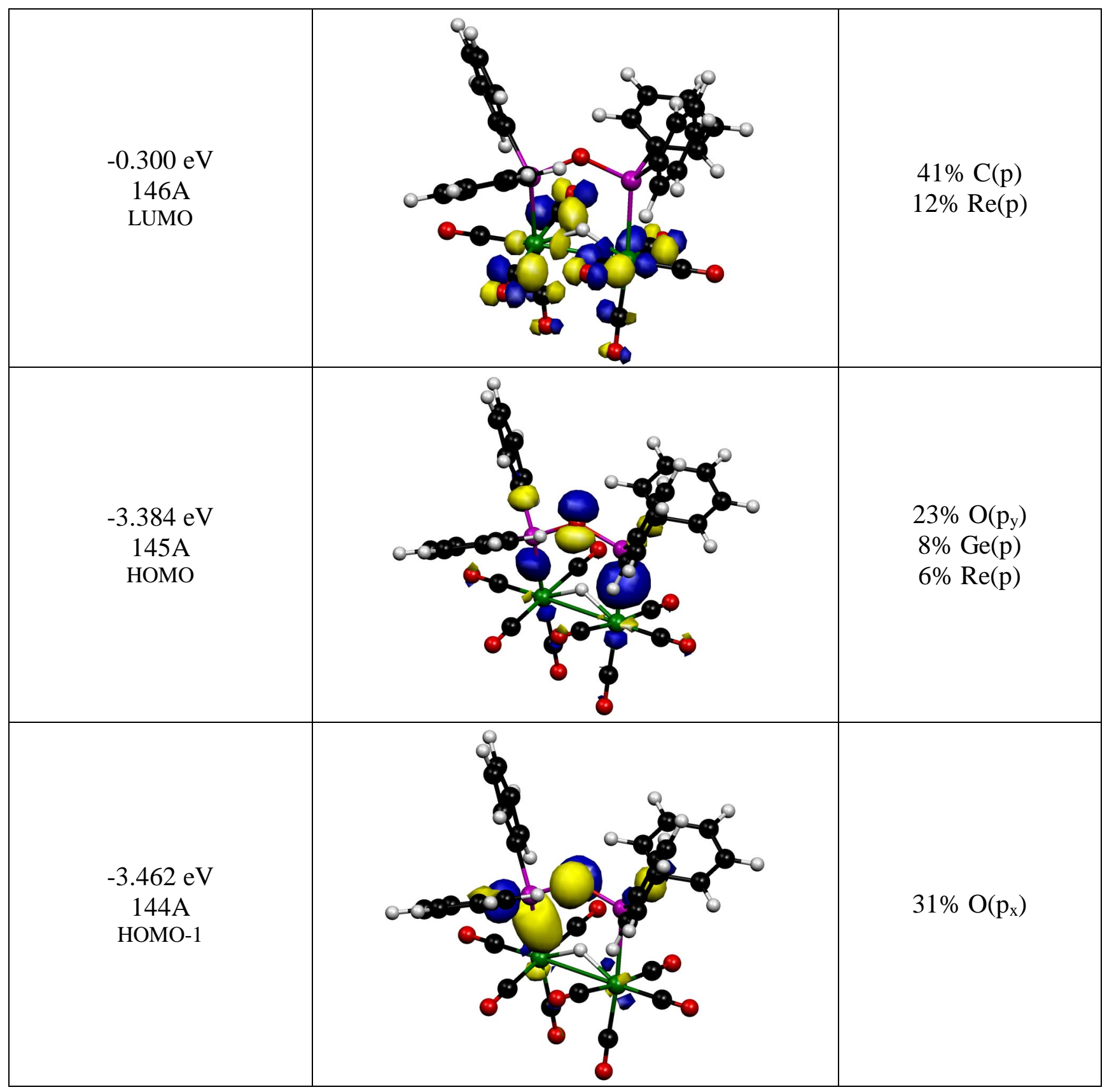




(143A




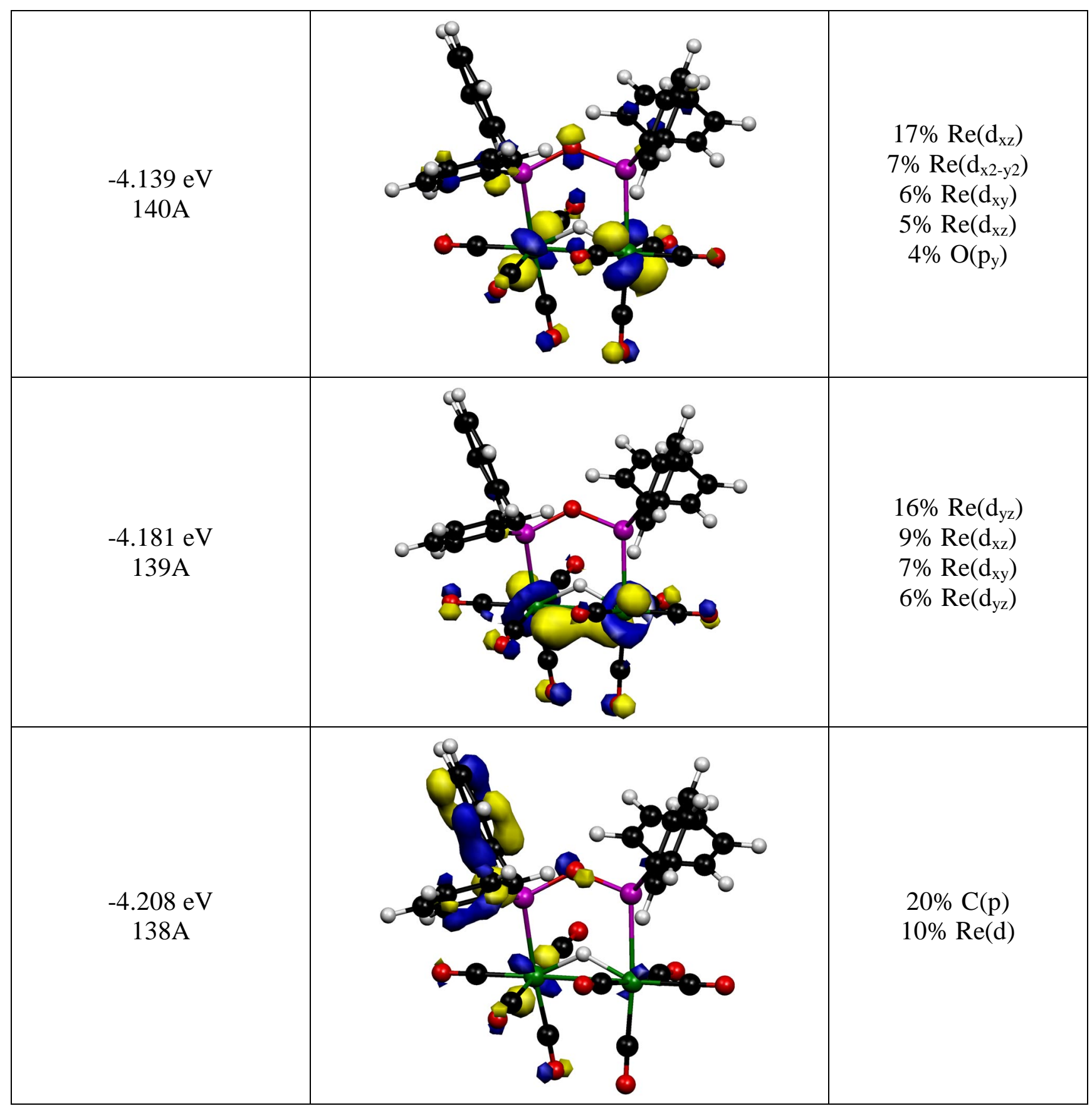




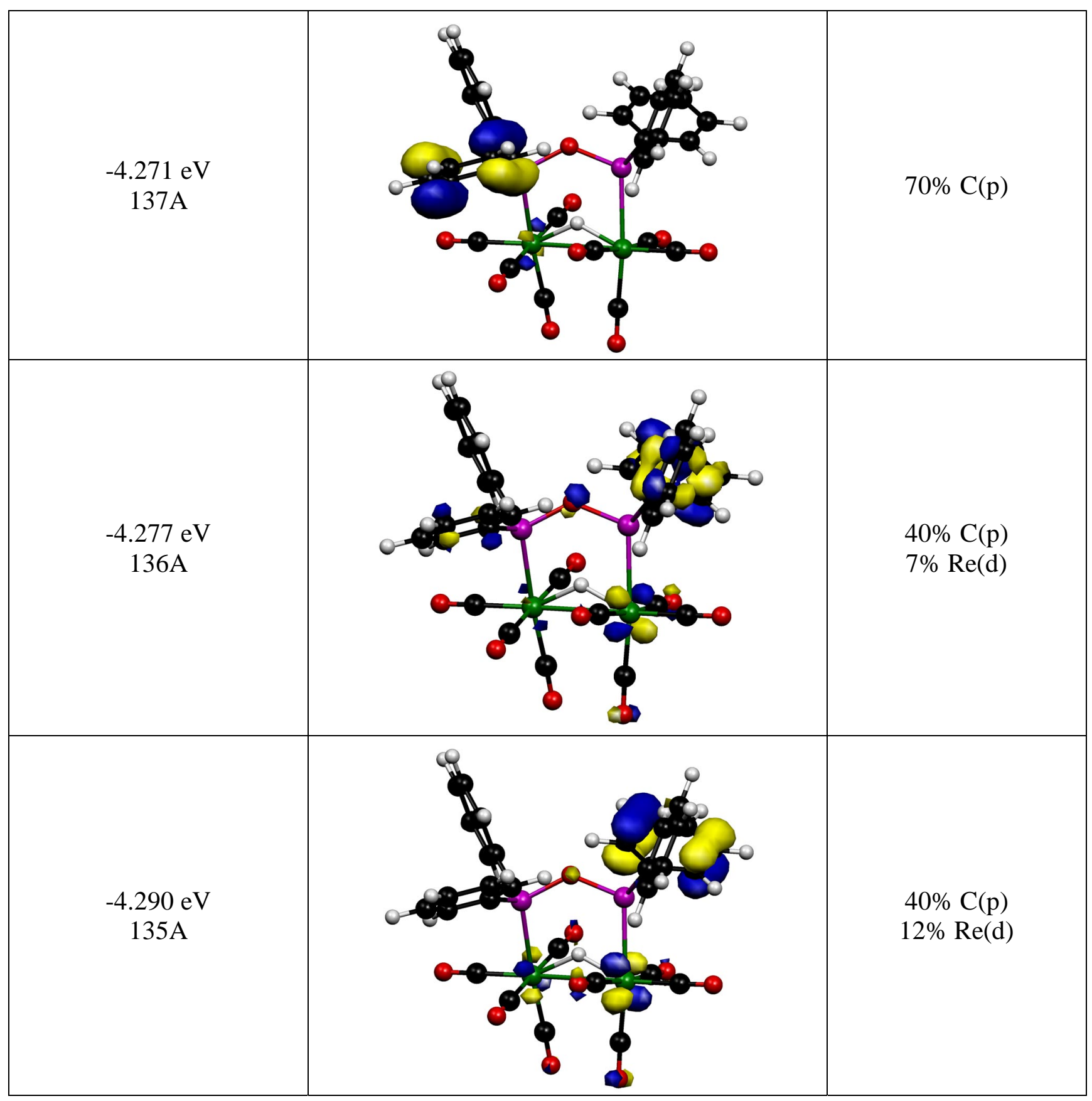




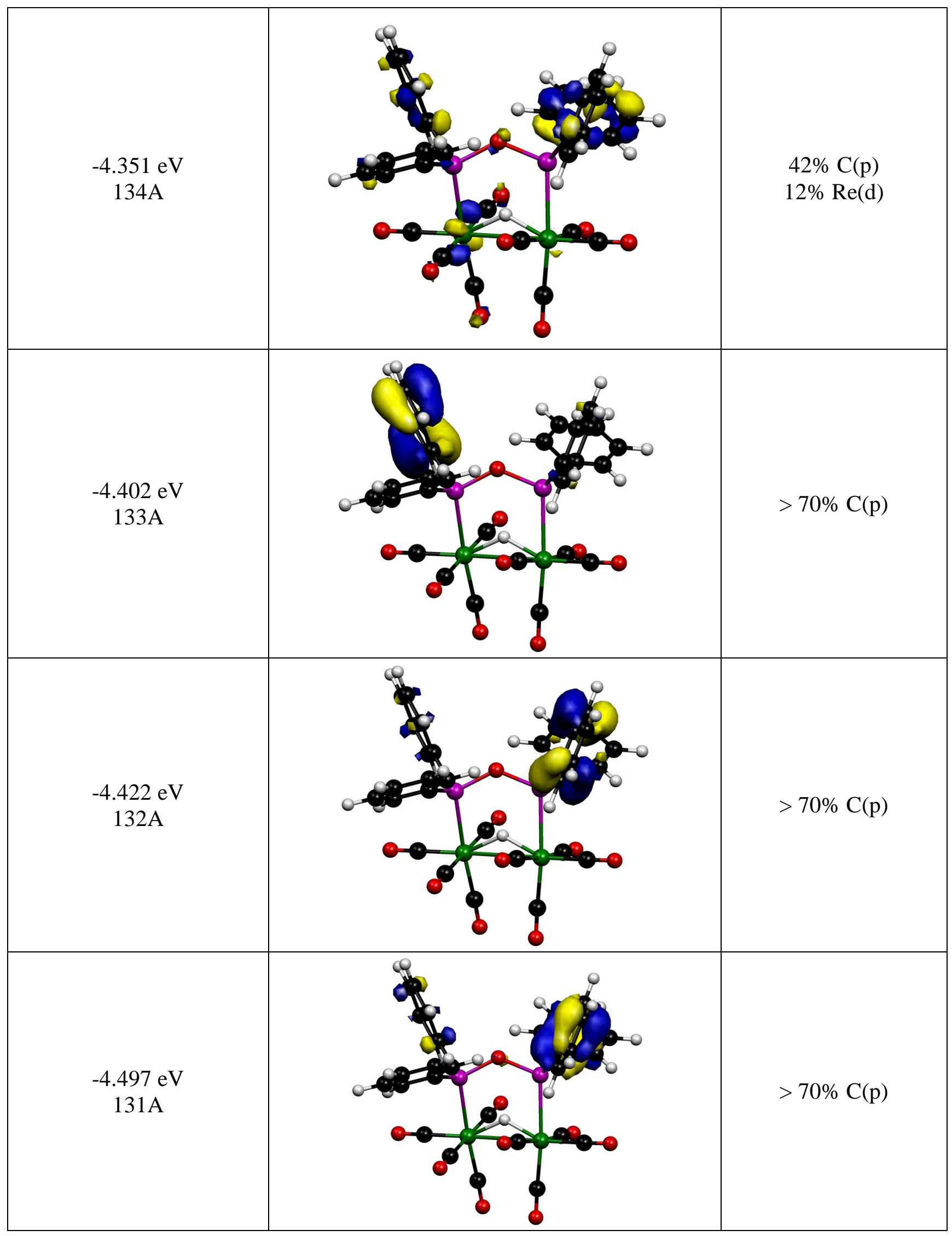




(130A


I. DFT Optimized Atomic Coordinates (ADF 2004.01, PW91, $C_{1}$ ) for 5 (xyz format)

II. DFT Optimized Atomic Coordinates (ADF 2004.01, PW91, $C_{1}$ ) for 4 (xyz format) 


\section{DFT Optimized Atomic Coordinates (ADF 2004.01, PW91, $C_{1}$ ) for 5 (xyz format)}

\begin{tabular}{|c|c|c|c|}
\hline $1 \mathrm{Re}$ & 0.554548 & -2.097270 & -1.614164 \\
\hline $2 \mathrm{Re}$ & 0.497917 & -2.065627 & 1.700576 \\
\hline $3 \mathrm{Ge}$ & -0.603287 & 0.278504 & -1.566719 \\
\hline $4 \mathrm{Ge}$ & 0.359108 & 0.576274 & 1.572480 \\
\hline 50 & 0.502804 & -2.176004 & -4.710234 \\
\hline 60 & 3.403769 & -0.765138 & -1.627298 \\
\hline 70 & 1.832903 & -4.932235 & -1.409106 \\
\hline 80 & -2.317340 & -3.358057 & -1.368455 \\
\hline 90 & -0.363988 & 1.186364 & 0.008032 \\
\hline $10 \mathrm{O}$ & 0.683367 & -5.174258 & 1.561266 \\
\hline $11 \mathrm{O}$ & -2.642208 & -2.160025 & 1.595824 \\
\hline $12 \mathrm{O}$ & 3.627850 & -1.780403 & 1.583375 \\
\hline $13 \mathrm{O}$ & 0.514838 & -1.991604 & 4.797122 \\
\hline $14 \mathrm{C}$ & 0.712513 & 3.723364 & -4.657409 \\
\hline $15 \mathrm{C}$ & 1.515713 & 2.595525 & -4.508969 \\
\hline $16 \mathrm{C}$ & -0.466292 & 3.828245 & -3.915960 \\
\hline $17 \mathrm{C}$ & 1.136869 & 1.581801 & -3.626138 \\
\hline $18 \mathrm{C}$ & 0.509395 & -2.142411 & -3.543886 \\
\hline $19 \mathrm{C}$ & -0.834565 & 2.812368 & -3.035689 \\
\hline $20 \mathrm{C}$ & -4.661129 & -0.042783 & -2.905895 \\
\hline $21 \mathrm{C}$ & -3.269677 & -0.080791 & -2.844378 \\
\hline $22 \mathrm{C}$ & -0.037737 & 1.672921 & -2.878207 \\
\hline $23 \mathrm{C}$ & -5.394341 & 0.400952 & -1.806186 \\
\hline $24 \mathrm{C}$ & -2.590481 & 0.330074 & -1.692415 \\
\hline $25 \mathrm{C}$ & 2.348810 & -1.241933 & -1.621446 \\
\hline $26 \mathrm{C}$ & 1.346469 & -3.876084 & -1.452741 \\
\hline $27 \mathrm{C}$ & -1.270392 & -2.876622 & -1.461712 \\
\hline $28 \mathrm{C}$ & -4.729036 & 0.816505 & -0.654396 \\
\hline $29 \mathrm{C}$ & -3.336870 & 0.782930 & -0.599975 \\
\hline $30 \mathrm{C}$ & 3.783200 & 2.835620 & 0.426526 \\
\hline $31 \mathrm{C}$ & 2.539006 & 2.210969 & 0.456815 \\
\hline $32 \mathrm{C}$ & 4.607655 & 2.801708 & 1.550879 \\
\hline $33 \mathrm{C}$ & 0.623113 & -4.011984 & 1.581071 \\
\hline $34 \mathrm{C}$ & 2.104363 & 1.532672 & 1.599141 \\
\hline $35 \mathrm{C}$ & -1.485570 & -2.106933 & 1.633209 \\
\hline $36 \mathrm{C}$ & 2.476348 & -1.872139 & 1.631959 \\
\hline $37 \mathrm{C}$ & 4.180130 & 2.139184 & 2.702936 \\
\hline $38 \mathrm{C}$ & 2.939708 & 1.504506 & 2.720888 \\
\hline $39 \mathrm{C}$ & -0.754628 & 1.562975 & 2.900264 \\
\hline $40 \mathrm{C}$ & -0.420788 & 2.879988 & 3.230616 \\
\hline $41 \mathrm{C}$ & -1.896941 & 1.002415 & 3.479089 \\
\hline $42 \mathrm{C}$ & 0.496030 & -2.030043 & 3.631969 \\
\hline $43 \mathrm{C}$ & -1.215794 & 3.625040 & 4.124315 \\
\hline $44 \mathrm{C}$ & -2.693506 & 1.733919 & 4.359297 \\
\hline $45 \mathrm{C}$ & -2.352598 & 3.046688 & 4.682956 \\
\hline $46 \mathrm{H}$ & 0.999696 & 4.519305 & -5.348892 \\
\hline $47 \mathrm{H}$ & 2.440462 & 2.499627 & -5.082954 \\
\hline $48 \mathrm{H}$ & -1.103958 & 4.709094 & -4.026199 \\
\hline $49 \mathrm{H}$ & -5.177714 & -0.358098 & -3.815563 \\
\hline $50 \mathrm{H}$ & -2.710329 & -0.431550 & -3.713555 \\
\hline $51 \mathrm{H}$ & 1.780348 & 0.705522 & -3.527100 \\
\hline $52 \mathrm{H}$ & -1.761815 & 2.909151 & -2.465229 \\
\hline $53 \mathrm{H}$ & -6.485704 & $4 \quad 0.430281$ & -1.850742 \\
\hline $54 \mathrm{H}$ & 4.109247 & 3.349458 & -0.477773 \\
\hline $55 \mathrm{H}$ & 1.885067 & 2.254924 & -0.415301 \\
\hline $56 \mathrm{H}$ & -5.295639 & 1.170625 & 0.208342 \\
\hline $57 \mathrm{H}$ & 0.451793 & -1.329646 & 0.036579 \\
\hline $58 \mathrm{H}$ & -2.813902 & 1.126816 & 0.293611 \\
\hline $59 \mathrm{H}$ & 5.586417 & 3.287600 & 1.530920 \\
\hline $60 \mathrm{H}$ & 0.467037 & 3.346012 & 2.794354 \\
\hline $61 \mathrm{H}$ & 4.821766 & 2.112810 & 3.587012 \\
\hline $62 \mathrm{H}$ & -2.182519 & -0.022782 & 3.243943 \\
\hline $63 \mathrm{H}$ & 2.623841 & 0.982916 & 3.625989 \\
\hline $64 \mathrm{H}$ & -0.939988 & 4.653336 & 4.369616 \\
\hline $65 \mathrm{H}$ & -3.584262 & 1.275223 & 4.795938 \\
\hline $66 \mathrm{H}$ & -2.976475 & 3.618728 & 5.373582 \\
\hline
\end{tabular}




\section{DFT Optimized Atomic Coordinates (ADF 2004.01, PW91, $C_{1}$ ) for 4 (xyz format)}

\begin{tabular}{|c|c|c|c|}
\hline $1 \mathrm{Re}$ & 0.569069 & -2.068150 & -1.611857 \\
\hline $2 \mathrm{Re}$ & 0.483474 & -2.059345 & 1.710919 \\
\hline $3 \mathrm{Ge}$ & -0.671224 & 0.197454 & -1.739659 \\
\hline $4 \mathrm{Ge}$ & 0.455487 & 0.526580 & 1.749906 \\
\hline $5 \mathrm{O}$ & 0.481784 & -2.209206 & -4.711614 \\
\hline 60 & 3.362967 & -0.623777 & -1.607258 \\
\hline $7 \mathrm{O}$ & 2.023898 & -4.814525 & -1.350861 \\
\hline $8 \mathrm{O}$ & -2.231741 & -3.483827 & -1.344175 \\
\hline $9 \mathrm{O}$ & -0.320153 & 1.111294 & 0.008234 \\
\hline $10 \mathrm{O}$ & 0.541421 & -5.168106 & 1.397100 \\
\hline $11 \mathrm{O}$ & -2.658800 & -2.043844 & 1.584577 \\
\hline $12 \mathrm{O}$ & 3.631331 & -1.929897 & 1.648562 \\
\hline $13 \mathrm{O}$ & 0.460443 & -2.006634 & 4.808447 \\
\hline $14 \mathrm{C}$ & 0.703040 & 3.727343 & -4.669364 \\
\hline $15 \mathrm{C}$ & 1.504790 & 2.598620 & -4.515204 \\
\hline $16 \mathrm{C}$ & -0.501903 & 3.822808 & -3.955527 \\
\hline $17 \mathrm{C}$ & 1.106357 & 1.577227 & -3.652875 \\
\hline $18 \mathrm{C}$ & 0.519710 & -2.165417 & -3.552163 \\
\hline $19 \mathrm{C}$ & -0.894589 & 2.791985 & -3.103347 \\
\hline $20 \mathrm{C}$ & -4.731577 & 0.041096 & -2.907621 \\
\hline $21 \mathrm{C}$ & -3.342478 & -0.021555 & -2.866788 \\
\hline $22 \mathrm{C}$ & -0.092362 & 1.658107 & -2.937121 \\
\hline $23 \mathrm{C}$ & -5.444471 & 0.471227 & -1.782489 \\
\hline $24 \mathrm{C}$ & -2.643691 & & -1.707078 \\
\hline $25 \mathrm{C}$ & 2.331958 & -1.143404 & -1.622175 \\
\hline $26 \mathrm{C}$ & 1.478219 & -3.795988 & -1.422449 \\
\hline $27 \mathrm{C}$ & -1.210453 & -2.957708 & -1.442172 \\
\hline $28 \mathrm{C}$ & -4.757487 & 0.827780 & -0.625757 \\
\hline $29 \mathrm{C}$ & -3.365653 & 0.754127 & -0.585889 \\
\hline $30 \mathrm{C}$ & 3.817020 & 2.764802 & 0.371800 \\
\hline $31 \mathrm{C}$ & 2.575548 & 2.139454 & 0.443029 \\
\hline $32 \mathrm{C}$ & 4.644424 & 2.797560 & 1.491575 \\
\hline $33 \mathrm{C}$ & & -4.018316 & 1.534856 \\
\hline $34 \mathrm{C}$ & 2.146949 & 1.537889 & 1.630007 \\
\hline $35 \mathrm{C}$ & -1.504348 & -2.048096 & 1.628531 \\
\hline $36 \mathrm{C}$ & 2.479658 & -1.975141 & 1.670033 \\
\hline $37 \mathrm{C}$ & 4.226384 & 2.204673 & \\
\hline $38 \mathrm{C}$ & 2.985455 & 1.575904 & 2.750502 \\
\hline $39 \mathrm{C}$ & -0.733670 & 1.579823 & 2.925608 \\
\hline $40 \mathrm{C}$ & -0.403684 & 2.899259 & 3.259183 \\
\hline $41 \mathrm{C}$ & -1.891062 & 1.017675 & 3.474692 \\
\hline $42 \mathrm{C}$ & 0.464469 & -2.050056 & 3.648656 \\
\hline $43 \mathrm{C}$ & -1.222901 & 3.643770 & 4.131489 \\
\hline $44 \mathrm{C}$ & -2.706117 & 1.747077 & 4.336072 \\
\hline $45 \mathrm{C}$ & -2.372257 & 3.059199 & 4.667035 \\
\hline $46 \mathrm{H}$ & 1.005044 & 4.529824 & -5.345963 \\
\hline $47 \mathrm{H}$ & 2.440110 & 2.506391 & -5.072919 \\
\hline $48 \mathrm{H}$ & -1.137146 & 4.703947 & -4.076108 \\
\hline $49 \mathrm{H}$ & -5.266229 & -0.242165 & -3.816445 \\
\hline $50 \mathrm{H}$ & -2.802070 & -0.354179 & -3.755007 \\
\hline $51 \mathrm{H}$ & 1.745850 & 0.698986 & -3.553116 \\
\hline $52 \mathrm{H}$ & -1.852029 & 2.870186 & -2.579966 \\
\hline $53 \mathrm{H}$ & -6.535634 & 0.527409 & -1.815133 \\
\hline $54 \mathrm{H}$ & 4.140778 & 3.225204 & -0.561458 \\
\hline $55 \mathrm{H}$ & 1.937776 & 2.122992 & -0.440984 \\
\hline $56 \mathrm{H}$ & -5.304867 & 1.163065 & 0.256217 \\
\hline $57 \mathrm{H}$ & 0.465878 & -1.319309 & 0.042430 \\
\hline $58 \mathrm{H}$ & -0.690722 & 2.019699 & 0.059789 \\
\hline $59 \mathrm{H}$ & -2.850505 & 1.023630 & 0.336179 \\
\hline $60 \mathrm{H}$ & 5.623056 & 3.280840 & 1.434126 \\
\hline $61 \mathrm{H}$ & 0.504148 & 3.357502 & 2.857416 \\
\hline $62 \mathrm{H}$ & 4.872472 & 2.226371 & 3.563804 \\
\hline $63 \mathrm{H}$ & -2.167492 & -0.010972 & 3.241880 \\
\hline $64 \mathrm{H}$ & 2.681089 & 1.113989 & 3.691519 \\
\hline $65 \mathrm{H}$ & -0.952334 & 4.669717 & 4.390815 \\
\hline $66 \mathrm{H}$ & -3.601528 & 1.286714 & 4.759995 \\
\hline $67 \mathrm{H}$ & -3.005976 & 3.628208 & 5.351638 \\
\hline
\end{tabular}

